\title{
Prediction of Low-Velocity Face-on Impact Response of Composite Laminates using High-Fidelity Finite Element Modeling Techniques
}

\author{
Solver I. Thorsson ${ }^{1}$ \\ University of Michigan, Ann Arbor, MI, 48109-2140, USA \\ Akinori Yoshimura² \\ Aeronautical Technology Directorate, Japan Aerospace Exploration Agency (JAXA), 6-13-1 Osawa, Mitaka, Tokyo \\ 181-0015, JAPAN \\ Anthony M. Waas ${ }^{3}$ \\ University of Washington, Seattle, WA 98195-2400 USA \\ Mostafa Rassaian ${ }^{4}$ \\ Boeing Company, Seattle, WA, 98124, USA
}

\begin{abstract}
In this paper the response of a polymer matrix composite (PMC) laminate subjected to low-velocity face-on impact is studied both experimentally and numerically. Experiments for face-on impact were carried out on a laminated composite specimen, and post impact inspection of the specimen was done using ultrasound techniques to obtain the damage extent through the thickness of the laminate. Two high-fidelity finite element (FE) models have been developed to capture the response and failure mechanisms seen in the experiments. The University of Washington (UW) model utilizes Enhanced Schapery Theory (EST) to capture the non-linearity due to matrix micro cracking as well as macro intra-lamina matrix cracking and fiber failure. Discrete Cohesive Zone Model (DCZM) is implemented to capture the interlamina failure initiatiation and propagation. An exisiting model from The Japan Aerospace Exploration Agency (JAXA), was extended to include mesh objectivity and this extended model, referred to as the enhanced continuum damage mechanics (ECDM) model, was used for predicting intra-lamina pre-peak nonlinearity as well as matrix cracking and fiber failure. The ECDM model utilizes commercially available cohesive methods to capture inter-lamina damage and failure. The commercial finite element code, ABAQUS Explicit, was used in combination with user defined material and element subroutines to implement these different modeling strategies.
\end{abstract}

\section{Introduction}

$\mathrm{C}$ omposite laminates are widely used in many industry applications because they offer an attractive strength to weight ratio as well as giving the ability of tailoring the response of the structure according to each application. Once the structure is in service they are likely to be subjected to impact from many different sources, such as bird impact, collision with another vehicle or tool drops during maintenance, to name a few. The last example given belongs to the category of low-velocity (or low-energy) impacts. Low velocity impacts at low energy levels on composite structures can leave negligible visible damage on the surface of the structure. However,

Copyright@ 2016 by Solver I. Thorsson, Akinori Yoshimura, Anthony M. Waas and Mostafa Rassaian. Published with permission by the American Institute of Aeronautics and Astronautics.

${ }^{1}$ Graduate Student Research Assistant, Department of Aerospace Engineering

${ }^{2}$ Visiting Research Scientist, William E. Boeing Department of Aeronautics and Astronautics

${ }^{3}$ Boeing Egtvedt Endowed Professor of Aerostructures, Chairman, William E. Boeing Department of Aeronautics and Astronautics, AIAA Fellow

${ }^{4}$ Technical Fellow, Boeing Research and Technology, Seattle, WA, AIAA Associate Fellow

American Institute of Aeronautics and Astronautics 
when the structure is examined through the thickness with techniques such as ultrasound scanning, X-ray or microCT, delaminations, splitting and fiber fracture can be seen ${ }^{1,2}$. While the damage seems harmless on the surface, under cyclic loading it could grow and eventually lead to failure of the structure. When these damage mechanisms are activated in internal plies of the structure and cannot be detected by visual inspection, it is necessary to develop computational models to correctly identify the damage extent due to impact and furthermore the effect it has on the load carrying capability of the structure.

In this paper the response of a PMC laminate subjected to low-velocity face-on impact will be studied and the extent of internal damage will be vizualized using a ultrasound scanning method. Two different high-fidelity finite element (FE) models will be developed to predict the response, progressive damage and final damage state of the laminate. The different FE models will be compared against experimental results as well as being evaluated against each other.

\section{Experimental Results}

Face-on impact tests were performed at the Aeronautics and Astronautics department at the University of Washington. The impact tests were carried out on an Instron Dynatup 9200 drop tower system. The impact mass was chosen to be $7.5 \mathrm{~kg}$ and the impact energy 25 Joules $(6.7 \mathrm{~J}$ per $\mathrm{mm}$ of thickness, in accordance to ASTM D7136), resulting in a drop height and impact velocity of $0.34 \mathrm{~m}$ and $2.58 \mathrm{~m} / \mathrm{s}$, respectfully. A hemispherical impactor with a diameter of $20 \mathrm{~mm}$ was used. The specimen dimensions are $150 \mathrm{~mm}$ by $100 \mathrm{~mm}$. The specimen is simply supported in the form of rollers using a picture-frame boundary condition on both sides. The rollers are of diameter $6.35 \mathrm{~mm}$ and are placed $6.35 \mathrm{~mm}$ away from the edges of the specimen. The composite laminate material is IM7/8552 and the layup is $[0 / 45 / 0 / 90 / 0 /-45 / 0 / 45 / 0 /-45]_{\mathrm{s}}$, with the zero axis oriented in the longer direction of the specimen. Experimental and specimen information can be seen in table 1.

Two highspeed cameras (Photron SA-X) were used in a stereo setup to record the back surface of the specimen during impact. A mirror was placed below the specimen, orientated at $45^{\circ}$, to allow for the surface to be observed. Camera resolution was set to 768 pixels by 448 pixels and the recording speed was set to 25,000 frames per second, which results in roughly 175 images of the event. By applying black and white speckles to the back surface of the specimen we could utilize Digital Image Correlation (DIC) techniques to acquire the full-field strains on the back surface as well as the out-of-plane deflection during the impact event.

Impacts were carried out on multiple specimens to ensure repeatability, the experiments proved repeatable as can be seen in figure 1, where the load-time response of the laminates can be seen. From the load-time histories (figure 1) it can be gathered that a significant failure event occurs at around $5 \mathrm{kN}$ in all three experiments, from the back surface it can be seen that splitting initiates before the $5 \mathrm{kN}$ limit and keeps growing up until the peak load is reached. It is therefore clear that the failure event observed would be within the laminate in the form of sudden delamination growth, fiber breaking/rupture or a combination of both.

Post-impact inspection of the specimen with the naked eye showed very minimal damage. A slight dimple can be seen on the impacted side as well as a few cracks due to splitting on the back surface. Ultrasound scanning was used to visualize the damage extent through the thickness of the composites. The tested coupons were scanned on both sides, however, due to the nature of the method, the impacted side gave a better image of the overall damage due to the increasing damage area as the back surface is approached, see figure 3. Damage extent was estimated as an ellipse, and the average values for the major (2a) and minor (2b) axes were $39.6 \mathrm{~mm}$ and $26.0 \mathrm{~mm}$, respectively. The measurements for the three tests were all within $4-10 \%$ of each other as well as showing the same patterns.

Table 1: Impact and coupon specifications

\begin{tabular}{|l|l|}
\hline Impact Energy (Joules) & 25 \\
\hline Impact Mass (kg) & $7.5 \mathrm{~kg}$ \\
\hline Impactor: & Hemispherical, 20 mm diameter \\
\hline Material: & $\mathrm{IM} 7 / 8552$ \\
\hline Ply count: & 20 \\
\hline Layup: & {$[0 / 45 / 0 / 90 / 0 /-45 / 0 / 45 / 0 /-45]_{\mathrm{s}}$} \\
\hline Thickness [mm]: & 3.72 \\
\hline
\end{tabular}

2

American Institute of Aeronautics and Astronautics 


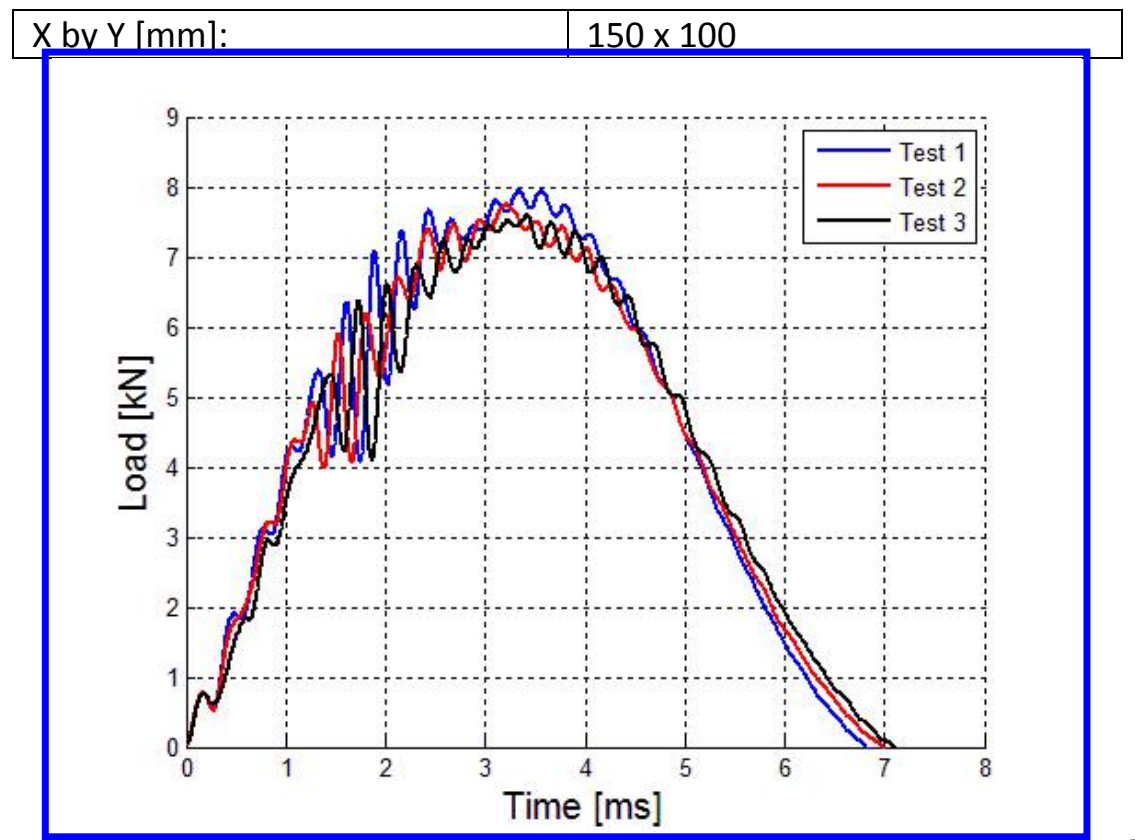

Figure 1: ExperimentaDoad curves for hoth of the impacts, OW in solid lines and ECDM in dotted lines

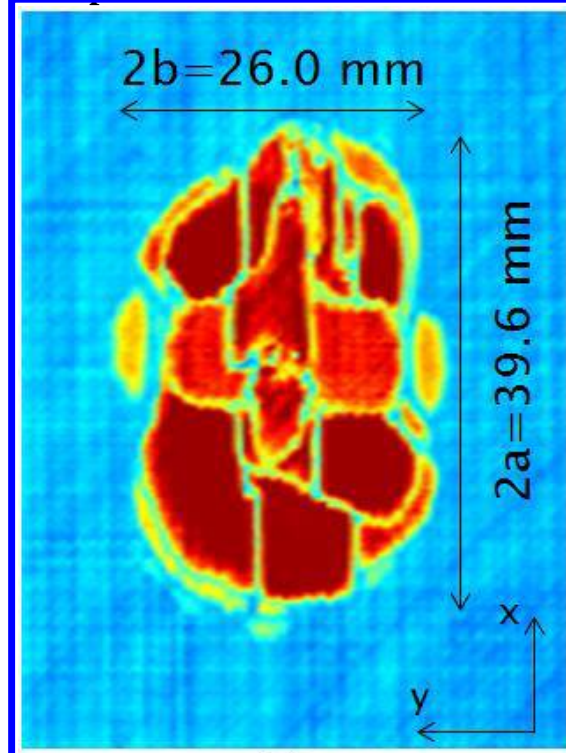

(a)

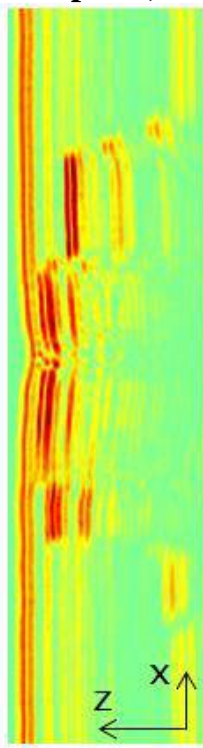

(b)

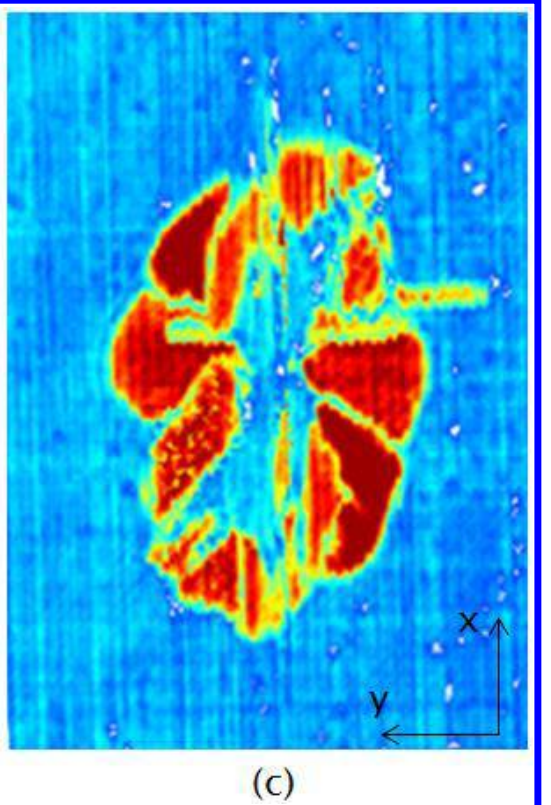

(c)

Figure 2: C-scans of the front surface (a) and back surface (c) and a B-scan vertical through the center of (a).

\section{Finite Element Modeling}

Two different FE models (figure 3) were used to model the impact response of the composite laminate. The two methods are then compared and evaluated against each other. FE analysis, in both models, was performed using the commercial FE code ABAQUS Explicit. Both models are lamina-level models where each lamina is modeled with 8 noded continuum shell elements with reduced integration (SC8R). The picture-frame supports were modeled as perfectly rigid as well as the impactor being modeled as a rigid body with a point mass $(7.5 \mathrm{~kg})$ and an initial velocity of $2.58 \mathrm{~m} / \mathrm{s}$ in the normal direction the the laminate. The contact between the supports and impactor with the laminate was modeled as Coulomb-type frictional contact with a frictional coefficient of 0.3. In the UW model each lamina is modeled with two separate continuum shell layers connected with tie constraints. The first layer is a rectangle of $75 \mathrm{~mm}$ by $50 \mathrm{~mm}$ with mesh size $0.5 \mathrm{~mm}$ by $0.5 \mathrm{~mm}$, the rectangle is centered underneath the impactor while the second layer models the rest of the coupon, which is a frame around the smaller rectangle, with a mesh size of $1.5 \mathrm{~mm}$ by $1.5 \mathrm{~mm}$. In the ECDM model, each lamina is modeled with a single layer with a fine mesh of 
$0.5 \mathrm{~mm}$ by $0.5 \mathrm{~mm}$ in a $60 \mathrm{~mm}$ by $40 \mathrm{~mm}$ area undearneath the impactor, while the mesh size size increases to $0.84 \mathrm{~mm}$ by $0.81 \mathrm{~mm}$ as it reaches the edges. As a result the number of continuum shell elements is 695,000 and 850,000 elements for the UW and ECDM model, respectfully.

The biggest difference with the models lies with the damage and failure modeling. Although the two models use similar modeling techniques, there is noticeable difference between the two. In the following sections a detailed description of each modeling technique will be given. The IM7/8552 material properties used were measured at the Universitv of Michigan. and these are shown in table 2

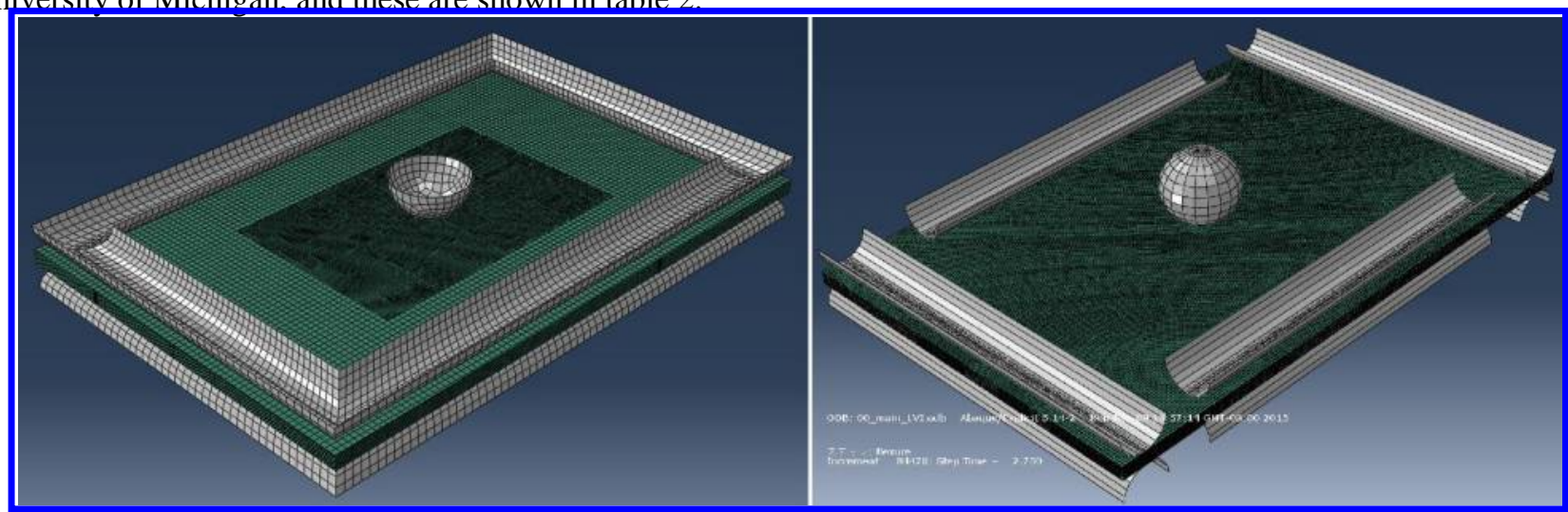

Figure 3: UW model (Left) and ECDM model (right)

\section{A. UW Model Description}

The UW model consists of Enhanced Schapery Theory (EST) ${ }^{3}$ and Discrete Cohesive Zone Model (DCZM) ${ }^{4}$ to capture failure mechanisms seen in the experiments. It is a continuation of modeling developed by $\mathrm{Ji}_{\text {et }} \mathrm{al}^{5}$. EST is a multiple-internal state variable (ISV) method capabable of modeling the intra-lamina damage and failure. It is a combination of Schapery Theory $(\mathrm{ST})^{6}$ and the Bazant-Oh Crack Band $(\mathrm{CB})$ model $^{7}$, see figure 4 . ST captures the non-linear material behavior due to matrix micro-cracking (which still preserves a positive tangent stiffness at the lamina scale) and CB can model the post peak failure regime where a macro-crack dissipates energy. Lamina failure includes shear and transverse macro-cracking (in the principal material frame) as well as fiber failure in compression and tension. The DCZM method is implemented for capturing the inter-lamina damage and failure due to delamations. EST is implemented using ABAQUS user material, VUMAT and DCZM is implemented using ABAQUS user elements, VUEL.

EST requires micro-damage functions to describe the pre-peak non-linear material behavior. These microdamage functions are backed out from experimental testing ${ }^{7}$. The shear $\left(g_{s}\right)$ and transverse $\left(e_{s}\right)$ microdamage functions can be seen in figure 5, and the compressive microdamage function was assumed the same as in tension. A strain based Hashin-Rotem criterion is used for matrix cracking and fiber failure. The failure strains and fracture toughnesses for each loading scenario is acquired from experimental data. The fracture properties are shown in table 2 .

For inter-lamina damage and failure modeling, DCZM elements are utilized. DCZM is a nodal tractionseparation method implemented using ABAQUS VUEL ${ }^{4}$. The element is a 8 noded element with 4 integration points, and three individual traction separation laws are used for the different directions of separation. These are, mode 1 for peel and mode 2 and mode 3 for shear, as shown in figure 6 . Triangular separation laws were used with a power-law mixed mode criterion with the power equal to 1

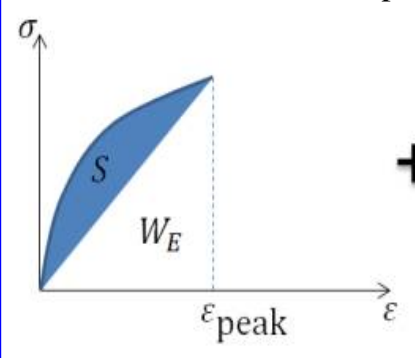

(a) Schapery theory

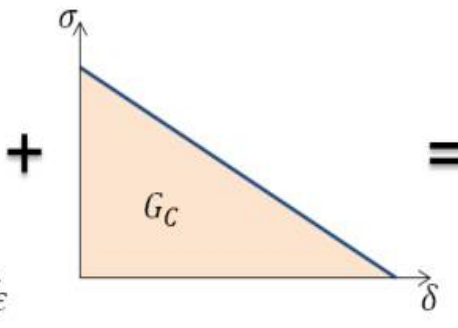

(b) Crack Band

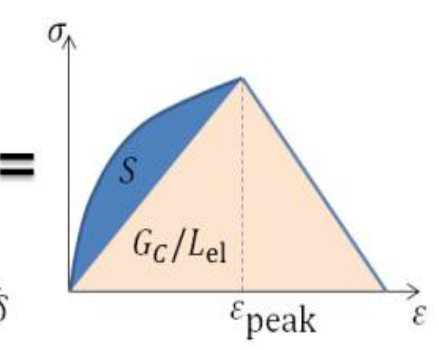

(c) Enhanced ST

Figure 4: Schematic explaining the implementation of Enhanced Schapery Theory 


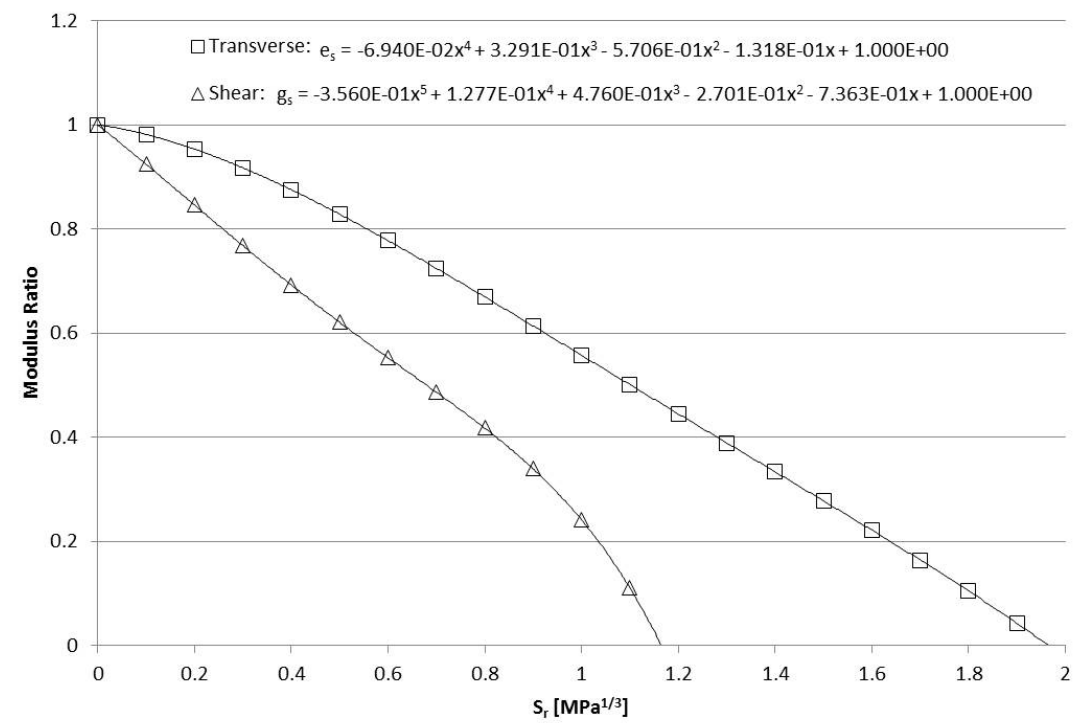

Figure 5: Schapery Theory micro damage functions
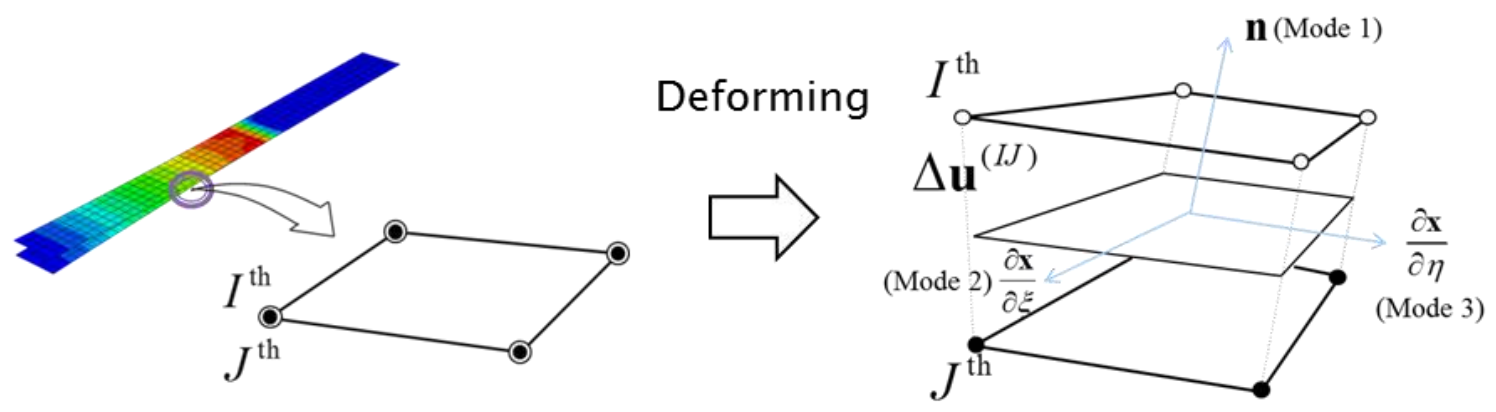

Figure 6: Schematic explaining the implementation of the Discrete Cohesive Zone Model element

\section{B. ECDM Model Description}

In the ECDM model, three types of damage are considered: in-plane fiber failure, in-plane cracks and delamination. Each type of damage was introduced in a separate manner. For fiber failure, simple stress criterion was used. For compression, when the fiber direction compressive stress $\sigma_{1}$ reached the compressive strength $S_{L}{ }^{C}$, the tangent stiffness in the fiber direction is suddenly reduced to zero. For tensile failure, after fiber direction tensile stress reached the tensile strength $S_{L}{ }^{T}$, stiffness was linearly reduced to zero using the smeared crack model (SCM) see figure 7. Note that $G_{f t}$ was divided by $l_{c}$ (characteristic length of the element) in order to match the dimensions. 


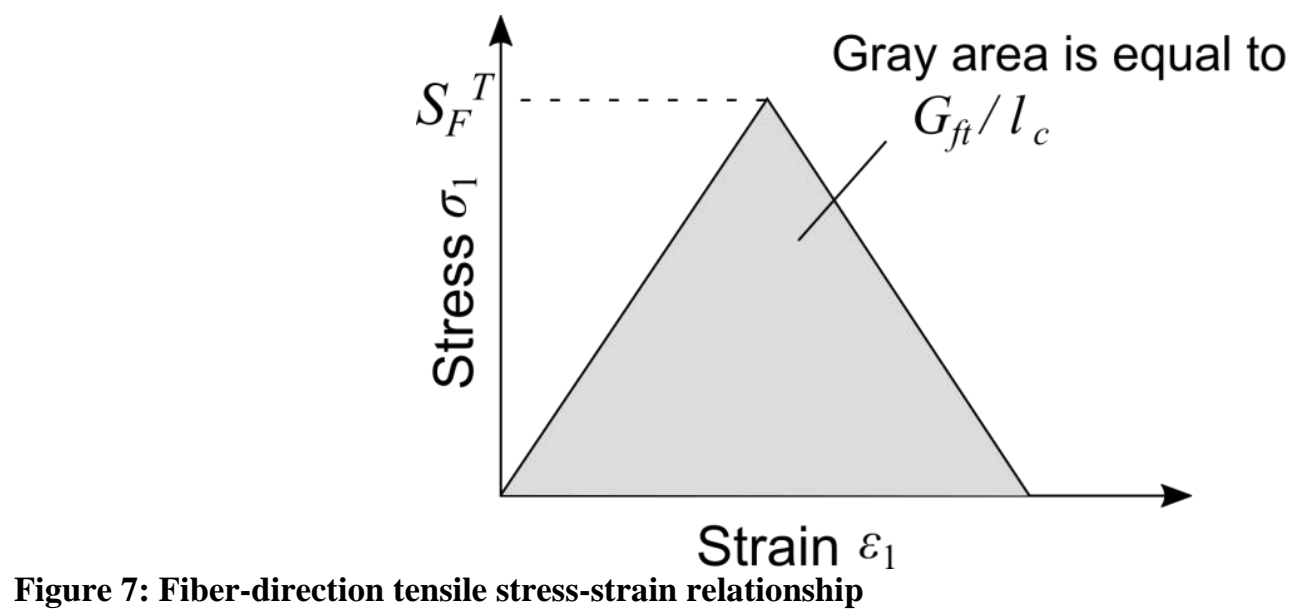

In-plane transverse and shear cracks are modeled using enhanced continuum damage mechanics (ECDM) model. In the model, pre-peak softening caused by the micro damage was modeled based on continuum damage mechanics (CDM), and post-peak behavior was modeled based on the smeared crack model (SCM). The transition from CDM to SCM is triggered by monitoring the stored strain energy in the element. When the stored strain energy starts to decrease SCM is initiated. In the CDM part, effective strain tensor $\tilde{\varepsilon}$ was introduced. The effective strain is calculated from the continuum strain tensor $\varepsilon$ as,

$$
\tilde{\boldsymbol{\varepsilon}}=\frac{1}{2}[(\boldsymbol{I}-\boldsymbol{D}) \boldsymbol{\varepsilon}+\boldsymbol{\varepsilon}(\boldsymbol{I}-\boldsymbol{D})]
$$

where $\boldsymbol{I}$ and $\boldsymbol{D}$ denote the second-order unit tensor and the damage tensor. In this study, the damage tensor $\boldsymbol{D}$ is defined by,

$$
\boldsymbol{D}=d_{C D M} \boldsymbol{n}_{2} \otimes \boldsymbol{n}_{2}
$$

where $\boldsymbol{n}_{2}$ denotes the unit vector in transverse direction (perpendicular to the fibers), and $d_{C D M}$ is a damage parameter. $d_{C D M}$ is initially equal to 0 (intact), and it gradually increases according to the damage evolution law discussed below. Using the effective strain, the stress tensor $\sigma$ is then calculated as,

$$
\sigma=C \tilde{\varepsilon}=\frac{1}{2} C[(I-D) \varepsilon+\varepsilon(I-D)]
$$

where $\boldsymbol{C}$ denotes the elastic stiffness tensor of the pristine material. Using eq. (3), stored strain energy per unit volume $e$ is calculated as,

$$
e=\frac{1}{2}\left\{C_{11} \varepsilon_{1}^{2}+\left(2-d_{C D M}\right) C_{12} \varepsilon_{1} \varepsilon_{2}+\left(1-d_{C D M}\right) C_{22} \varepsilon_{2}^{2}+\left(1-d_{C D M} / 2\right) C_{44} \gamma_{12}^{2}\right.
$$

where $C_{i j}$ denotes the $i j^{\text {th }}$ components of stiffness tensor. Note that continuum shell elements were used for lamina, so out-of-plane components were not included in eq. (4). Let $Y_{C D M}$ be the thermodynamic conjugate parameter corresponding to the damage parameter $d_{C D M} . Y_{C D M}$ can then be calculated by partially differentiating the specific elastic energy $e$ with respect to $d_{C D M}$ as,

$$
Y_{C D M}=-\frac{\partial e}{\partial d_{C D M}}=\frac{1}{2}\left[C_{12} \varepsilon_{1} \varepsilon_{2}+C_{22} \varepsilon_{2}^{2}+\frac{1}{2} C_{44} \gamma_{12}^{2}\right]
$$

In this study, the damage evolution law $d_{C D M}=f\left(Y_{C D M}\right)$ was determined by using static tensile test results of $\left[0 / 90_{3}\right]_{\mathrm{s}}$ CFRP specimens ${ }^{9}$ (see Fig. 8). 


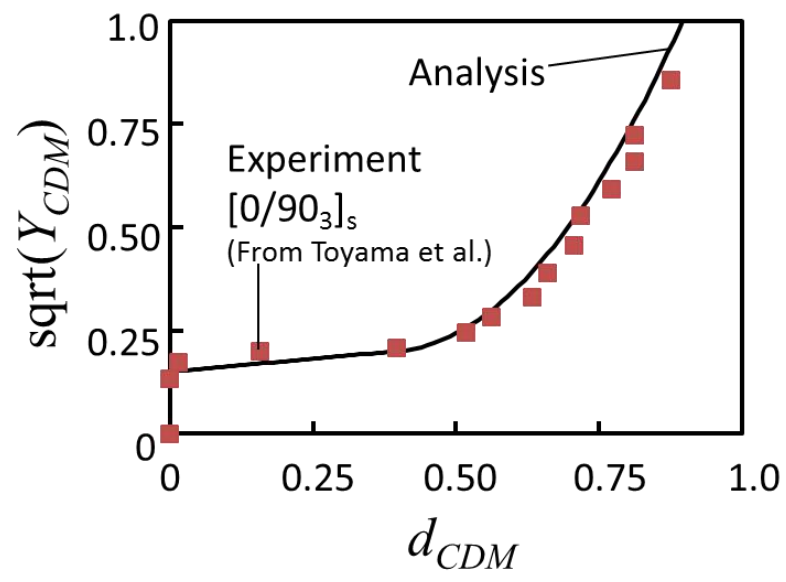

Figure 8: Damage evolution law (relationship between $d_{2}$ and $Y_{2}$ )

The post-peak response corresponding to a reduction in stress was calculated by using the SCM. In the SCM, transverse tensile and shear stress components $\sigma_{2}$ and $\tau_{12}$ are calculated from a traction-separation law. Dissipated energy by this element was set equal to the fracture toughness of the crack. Stress was calculated as follows. First, the mode mixity ratio was calculated. If peak values of stress are $\sigma^{0}{ }_{2}$ and $\tau^{0}{ }_{12}$, and corresponding strain components are $\varepsilon_{2}^{0}$ and $\gamma_{12}^{0}$, the mode mixity ratio was calculated as,

$$
m_{1}=\frac{\sigma_{2}^{0} \varepsilon_{2}^{0}}{2 e^{T}}, m_{2}=\frac{\tau_{12}^{0} \gamma_{12}^{0}}{2 e^{T}}
$$

where $e^{T}$ denotes total energy calculated as,

$$
e^{T}=\frac{1}{2}\left\{\sigma_{2}^{0} \varepsilon_{2}^{0}+\tau_{12}^{0} \gamma_{12}^{0}\right\}
$$

The total fracture toughness was calculated as,

$$
G_{C}=\frac{1}{\left\{\left(\frac{m_{1}}{G_{\mathrm{IC}}}\right)^{\alpha}+\left(\frac{m_{2}}{G_{\mathrm{IIC}}}\right)^{\alpha}\right\}^{\frac{1}{\alpha}}}
$$

where $G_{\text {Ic }}$ and $G_{\text {IIc }}$ denote mode I and mode II single-mode fracture toughnesses. $\alpha$ is parameter of power law. Each component of critical strain, where stress decreases, can be calculated as,

$$
\varepsilon_{2}^{f}=\frac{2 m_{1} G_{C}}{\sigma_{2}^{0} l_{c}}, \gamma_{12}^{f}=\frac{2 m_{2} G_{C}}{\tau_{12}^{0} l_{c}}
$$

where $l_{c}$ denotes characteristic length of the element. $l_{c}$ was calculated by dividing the volume of the element by the area of crack surface, so that the dissipated energy of the element is equal to that dissipated by the crack propoagation. In this model, effective strain was calculated as,

$$
\varepsilon=\sqrt{\varepsilon_{2}^{2}+\gamma_{12}^{2}}
$$

By using effective strain, the scalar damage parameter of SCM was calculated as,

American Institute of Aeronautics and Astronautics 


$$
d_{\mathrm{SCM}}=\frac{\varepsilon^{f}}{\varepsilon} \frac{\varepsilon-\varepsilon^{0}}{\varepsilon^{f}-\varepsilon^{0}}
$$

Where,

$$
\varepsilon^{f}=\sqrt{\varepsilon_{2}^{f 2}+\gamma_{12}^{f 2}}, \varepsilon^{0}=\sqrt{\varepsilon_{2}^{02}+\gamma_{12}^{02}}
$$

By using $d_{\mathrm{SCM}}$, stress was calculated as,

$$
\left\{\begin{array}{l}
\sigma_{1} \\
\sigma_{2} \\
\tau_{12}
\end{array}\right\}=\left[\begin{array}{ccc}
C_{11} & \left(1-d_{S C M}\right)\left(1-d_{C D M} / 2\right) C_{12} & 0 \\
\left(1-d_{S C M}\right)\left(1-d_{C D M} / 2\right) C_{12} & \left(1-d_{S C M}\right)\left(1-d_{C D M}\right) C_{22} & 0 \\
0 & 0 & \left(1-d_{S C M}\right)\left(1-d_{C D M} / 2\right) C_{44}
\end{array}\right]\left\{\begin{array}{l}
\varepsilon_{1} \\
\varepsilon_{2} \\
\gamma_{12}
\end{array}\right\}
$$

Equation (13) includes the damage parameter $d_{C D M}$ calculated using figure 8 in order to consider the effect of prepeak behavior. Note that after the peak, $d_{C D M}$ is not updated. Stress-strain relationship of a single element subjected to uniaxial tensile deformation is illustrated in Fig. 9. Fiber failure and transverse cracking were implemented in ABAQUS by using user subroutine VUMAT.

Delamination was modeled by introducing cohesive zone model in each interface, by using built-in surface based cohesive behavior in ABAQUS. In this method, cohesive traction is calculated based on the contact algorithm between two surfaces. Cohesive contact behavior was assigned at each interface of two adjacent laminae. Bilinear traction-separation law was utilized. Damage initiation was dictated by a stress quadratic criterion. For mixed mode fracture, power law was used. Material properties used in the calculation are summarized in Table 2.

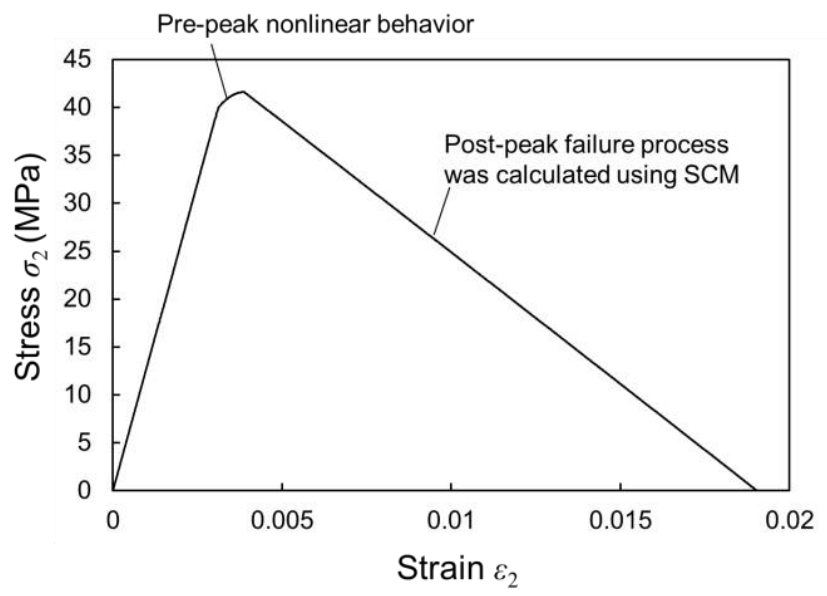

Figure 9: Single-element single-mode stress-strain relationship of ECDM model

\section{FE Model Results}

The load-time curve comparison and delamination growth can be seen in figure 10, and it is seen that the UW model predicts the pre-peak response of the experiment very well. The onset of failure is captured correctly in the form of compressive fiber failure in the upper layers of the laminate which then leads to fiber failure in tension in the lower layers. Delamination grows steadily during the impact as can be seen in figure 10. The damage accumulates during loading and follows the lower bound of the experimental response. The peak load is achieved a little later than in the experiment and therefore the over-all impact duration is longer than the experimental results show. The ECDM model also shows good agreement with experimental results, and the peak load is very similar to that of the experiments. The model shows a slightly different trend than the experiments as it is fairly linear up until the peak load. The model follows the upper bound of the experiments up until failure. Once the failure event happens, due to tensile fiber failure and unstable delamination growth, the load is reduced slightly below that of the experimental lower bounds and the impactor begins to rebound shortly after. The post-peak behavior is softer than 
that of the experiment. This is due to the residual stiffness of the laminate being under predicted and therefore the impactor is rebounding slower than it should. Delamination in the UW model (figure 11) is slightly over predicted. The trend of delaminations (petal shape) is captured to some extent. The UW model shows the delamination pattern changing depending on the angle-jump of the adjacent plies. Delamination jumps between interfaces can also be seen. The overall area of delamination in the ECDM model is under predicted when compared to the experimental data (figure 12). The model shows delamination size growing from the top layers to the bottom layers as well as the shape of damage area changing from circular to ellipsoidal. One reason for the over predicted delaminations in the UW model could be that the ultrasound scan gives a lower bound of the damage, and the resolution may not capture cracks that have closed up almost perfectly at the boundaries of the imaged delaminations.

Table 2: Input material properties (*) ECDM model only

\begin{tabular}{|c|c|}
\hline \multicolumn{2}{|l|}{ CFRP lamina } \\
\hline Longitudinal Young's modulus $E_{1}(\mathrm{MPa})$ & 154.46 \\
\hline Transverse Young's modulus $E_{2}(\mathrm{MPa})$ & 7.20 \\
\hline In-plane Poisson's ratio $v_{12}$ & 0.340 \\
\hline In-plane shear modulus $G_{12}(\mathrm{MPa})$ & 4.87 \\
\hline Density $\rho\left(\mathrm{g} / \mathrm{mm}^{3}\right)$ & 0.00157 \\
\hline Longitudinal Tensile Failure Strain $\mathrm{X}_{11}^{\mathrm{T}}(\%)$ & 1.47 \\
\hline Longitudinal Compressive Failure Strain $\mathrm{X}_{11}^{\mathrm{C}}(\%)$ & 1.31 \\
\hline Transverse Tensile Failure Strain $\mathrm{Y}_{22}^{\mathrm{T}}(\%)$ & 0.8 \\
\hline Transverse Compressive Failure Strain $\mathrm{Y}_{22} \mathrm{C}(\%)$ & 4.62 \\
\hline Shear Failure Strain $S_{12}(\%)$ & 2.27 \\
\hline Longitudinal tensile strength $S_{L}^{T}(\mathrm{MPa})(*)$ & 2271 \\
\hline Longitudinal compressive strength $S_{L}^{C}(\mathrm{MPa})(*)$ & 2023 \\
\hline Longitudinal tensile fracture toughness $G_{f}^{T}\left(\mathrm{Nmm} / \mathrm{mm}^{2}\right)$ & 40.0 \\
\hline Longitudinal compressive fracture toughness $G_{f}^{C}\left(\mathrm{Nmm} / \mathrm{mm}^{2}\right)$ & 10.0 \\
\hline Mode-I fracture toughness of transverse crack $G_{\mathrm{IC}}\left(\mathrm{Nmm} / \mathrm{mm}^{2}\right)$ & 0.384 \\
\hline Mode-II fracture toughness of transverse crack $G_{\text {IIC }}\left(\mathrm{Nmm} / \mathrm{mm}^{2}\right)$ & 2.184 \\
\hline \multicolumn{2}{|l|}{ Interface properties } \\
\hline Mode-I initial stiffness $k_{n}(\mathrm{MPa} / \mathrm{mm})$ & $2.0 \times 10^{4}$ \\
\hline Mode-II initial stiffness $k_{t}(\mathrm{MPa} / \mathrm{mm})$ & $2.0 \times 10^{4}$ \\
\hline Mode-III initial stiffness $k_{b}(\mathrm{MPa} / \mathrm{mm})$ & $2.0 \times 10^{4}$ \\
\hline Mode-I maximum traction $\sigma_{\max }(\mathrm{MPa})$ & 55.0 \\
\hline Mode-II maximum traction $\tau_{\max }(\mathrm{MPa})$ & 72.0 \\
\hline Mode-III maximum traction $\tau_{\max }(\mathrm{MPa})$ & 72.0 \\
\hline Mode-I fracture toughness $G_{\mathrm{IC}}\left(\mathrm{Nmm} / \mathrm{mm}^{2}\right)$ & 0.384 \\
\hline Mode-II fracture toughness $G_{\text {IIC }}\left(\mathrm{Nmm} / \mathrm{mm}^{2}\right)$ & 2.184 \\
\hline Mode-III fracture toughness $G_{\text {IIIC }}\left(\mathrm{Nmm} / \mathrm{mm}^{2}\right)$ & 2.184 \\
\hline
\end{tabular}




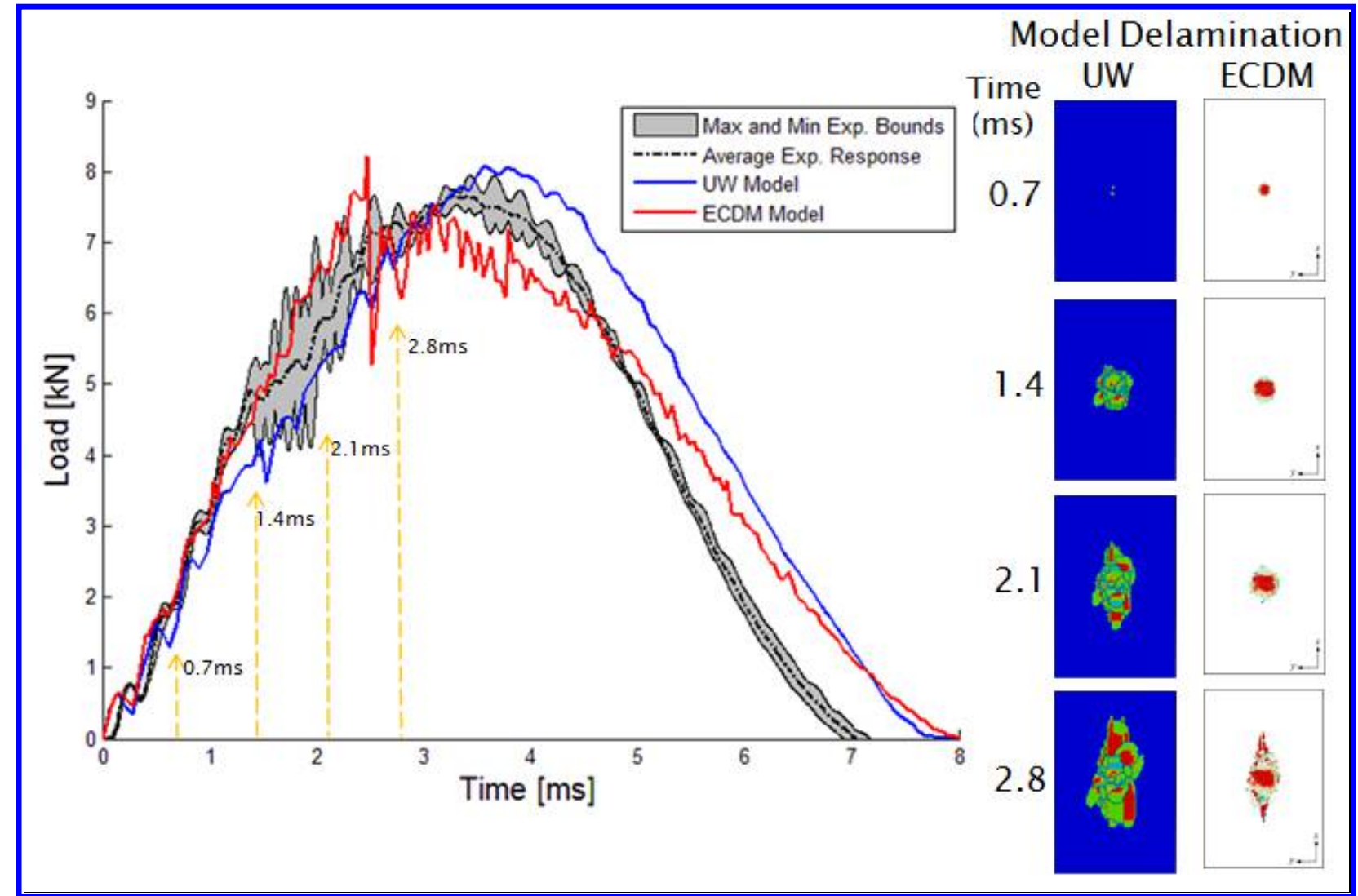

Figure 10: Comparison of load history between experimental and simulated results. Area marked in gray represents the maximum and minimum bounds of the experimental impact response and the dotted black line is the average response. Blue and red curves represent the UW and ECDM model, respectfully. On the right side the delamination growth can be seen, at $2.8 \mathrm{~ms}$ the delamination has reached the final state.

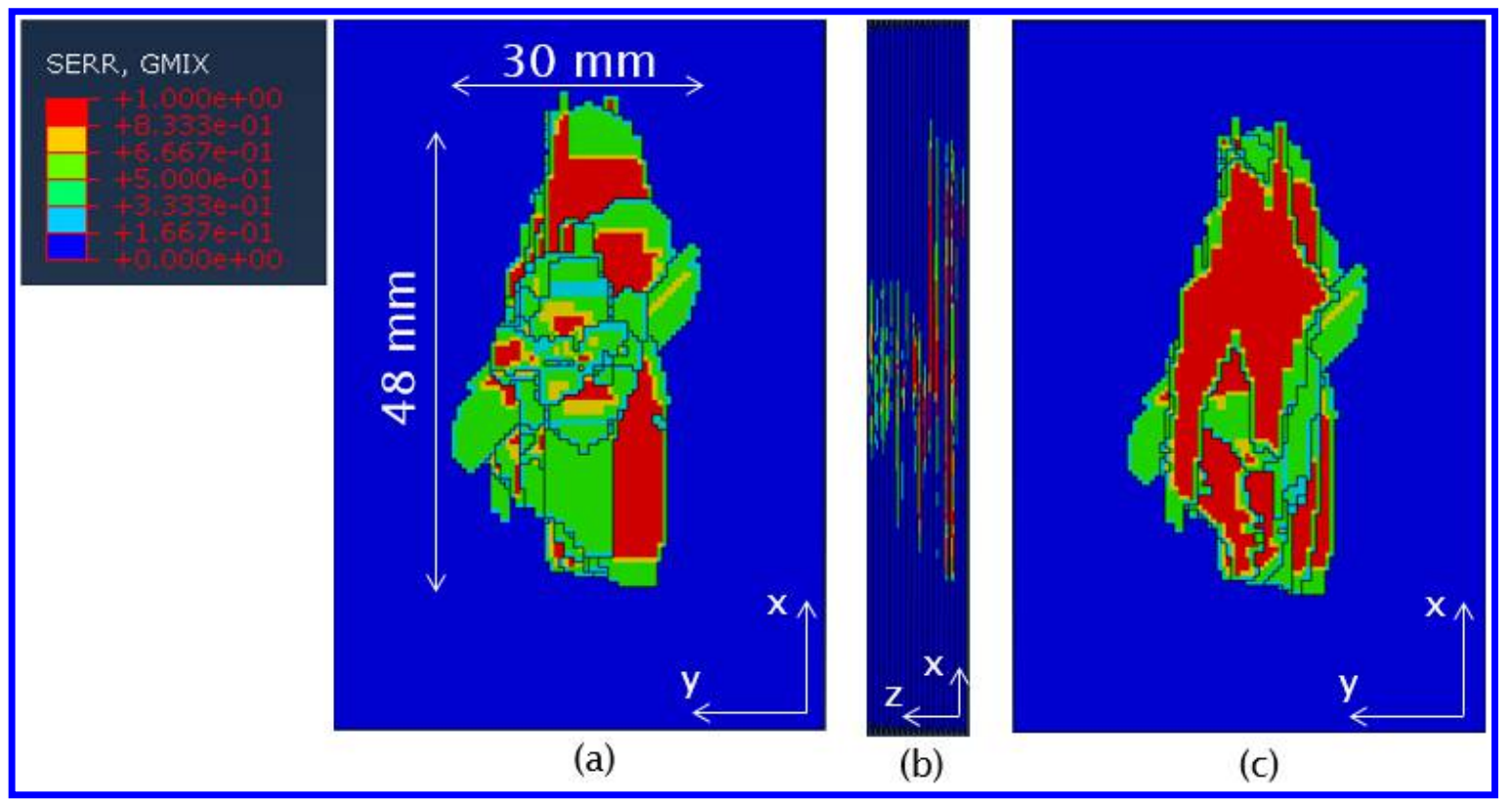

Figure 11: Delamination results for the UW model seen from the front (a), back (c) and through the thickness (b). Delaminations are predicted slightly larger than the damage seen in the ultrasound, the delamination pattern is captured to some extent. 


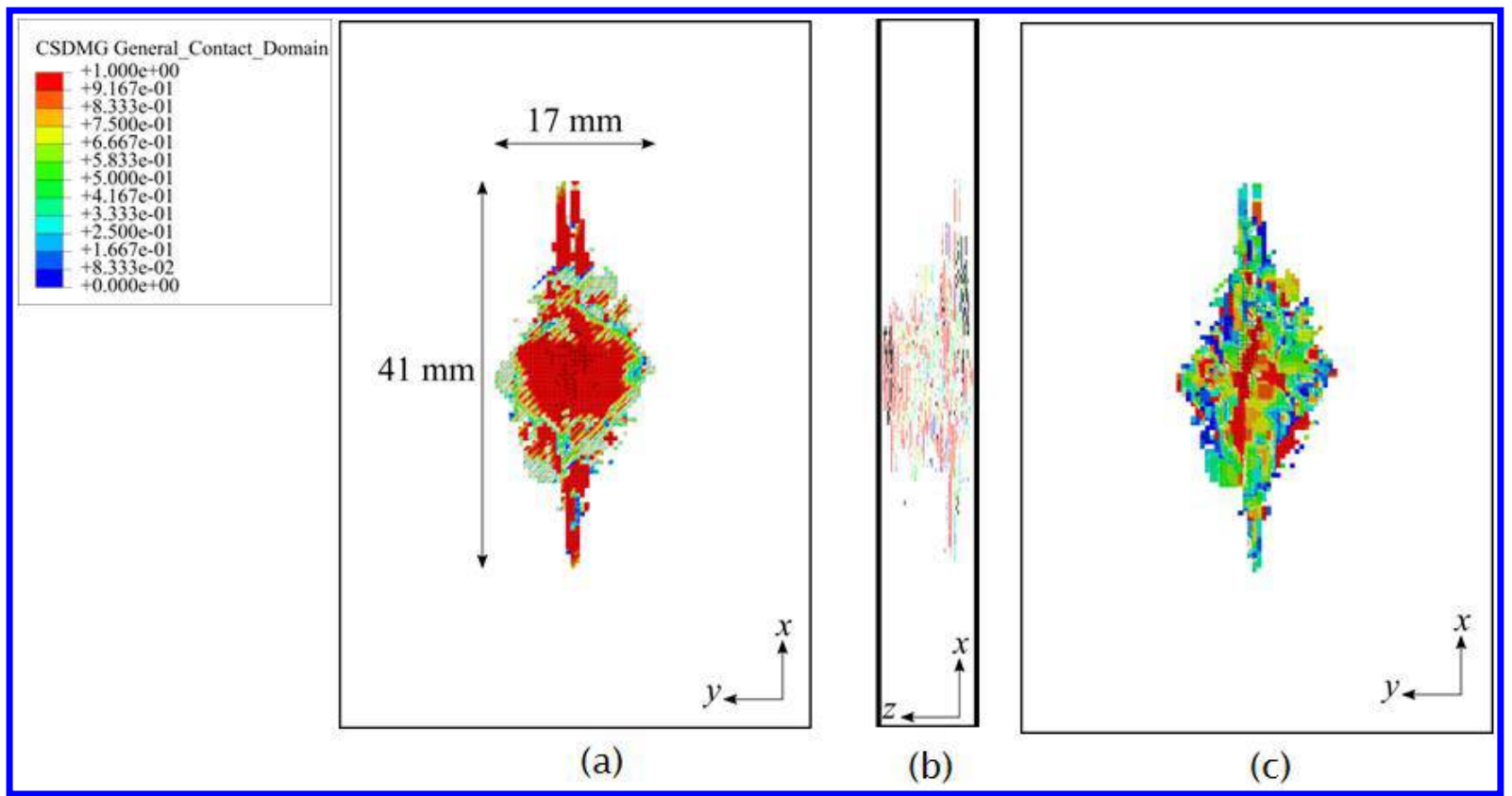

Figure 12: Delamination results for the ECDM model seen from the front (a), back (c) and through the thickness (b). Delaminations are under predicted.

\section{Conclusions}

Experimental and numerical results for a laminated composite subject to face-on impact has been presented. The extent of the impact damage through the thickness of the laminate has been observed. Two finite element models have been introduced to capture the impact response as well as the extent of damage. The two models show good agreement with experimental results. The UW model, consisting of EST and DCZM for in-plane and out-of-plane damage, respectively, captures the pre-peak loading response accurately while the total duration of the impact is over predicted. The overall extent of damage is slightly over predicted. The petal-shape of the delamination areas is captured to some extent. The ECDM model, which consists of ECDM for in-plane damage and failure, and Abaqus cohesive contact for out-of-plane failure, shows reasonable agreement with the experimental load-history curve. The ECDM model also over predicts the impact duration as well as the post-peak being softer than the experimental post-peak response. The ECDM model moderately under predicts the total extent of damage, the petal-shape that is seen in the experiments is not captured. Both models show a longer impact duration. This behavior is observed due to the residual bending stiffness of the impacted laminate not being captured correctly. When in the simulation an element has failed, it is either deleted or given a residual stiffness. Because of this, the post-peak response of the laminate can be incorrectly captured if the element is deleted or if the residual stiffness is too low compared to reality. Further investigation into the residual stiffness is required.

\section{Acknowledgements}

The authors are grateful for the support and encouragement from Steve Precup, Steve Wanthal, Salvatore Liguore, Brian Justusson, Joe Schafer and Gary Rennieri from the Boeing Company. The authors would like to thank Jaspar Marek from the University of Washington for his assistance.

\section{References}

${ }^{1}$ Razi, H., and Kobayashi., A. S., "Delamination in Cross-ply Laminated Composite Subjected to Low-velocity Impact," AIAA Journal, Vol. 31, No. 8, 1993, pp.1498-1502.

2Davies, G.A.O., and R. Olsson, "Impact on composite structures," Aeronautical Journal 108.1089 (2004): $541-563$.

'Pineda, E.J. and Waas, A.M., "Numerical implementation of a multiple-ISV thermodynamically-based work potential theory for modeling progressive damage and failure in fiber-reinforced laminates," International Journal of Fracture, Vol. 182, No. 1, 2013, pp. 93-122.

$\checkmark 4$ Xie, De, and Waas, A.M., "Discrete cohesive zone model for mixed-mode fracture using finite element analysis," Engineering Fracture Mechanics, 73.13 (2006): 1783-1796. 
${ }^{5}$ Ji, W., Sringeri, S.P., Thorsson, S., Kosztowny, C., Waas, A.M., Rassaian, M., Liguore, S. "Face-on and Edge-on Impact Response of Composite Laminates,” 2014.

-6Schapery, R.A., "A theory of mechanical behavior of elastic media with growing damage and other changes in structure," Journal of Mechanics and Physics of Solids, Vol. 38, No. 2, 1990, pp. 215-253.

${ }^{7}$ Bazant, Z.P. and Oh, B.H., “Crack band theory for fracture of concrete,' Materiaux et constructions, Vol. 16, No. 93, pp. 155-177.

${ }^{8}$ Heinrich, C. and Waas, A. M., "Investigation of progressive damage and fracture in laminated composites using the smeared crack approach," Computers, Materials \& Continua, Vol. 35, No.2, 2013.

>Toyama N, Noda J, Okabe T., "Quantitative damage detection in cross-ply laminates using Lamb wave method." Composites Science and Technology, Vol. 63, 2003, pp. 1473-1479 
This article has been cited by:

1. Solver I. Thorsson, Anthony M. Waas. 8.11 Foreign Object Impact on Composite Materials and the Modeling Challenges 206-218. [Crossref]

2. Solver I. Thorsson, Sunil P. Sringeri, Anthony M. Waas, Brian P. Justusson, Mostafa Rassaian. 2017. Experimental Investigation of Composite Laminates Subject to Low-Velocity Edge-on Impact and Compression After Impact. Composite Structures . [Crossref] 